\title{
Airborne Wind Profiling with the Data Acquisition and Processing System for a Pulsed 2-Micron Coherent Doppler Lidar System
}

\author{
Jeffrey Y. Beyon ${ }^{1 *}$, Grady J. Koch ${ }^{2}$, and Michael J. Kavaya ${ }^{3}$ \\ ${ }^{1}$ NASA Langley Research Center, MS 488, Hampton, VA 23681, USA, Jeffrey.Y.Beyon@nasa.gov \\ ${ }^{2}$ NASA Langley Research Center, MS 468, Hampton, VA 23681, USA, Grady.J.Koch@nasa.gov \\ ${ }^{3}$ NASA Langley Research Center, MS 468, Hampton, VA 23681, USA, Michael.J.Kavaya@nasa.gov
}

\begin{abstract}
A pulsed 2-micron coherent Doppler lidar system at NASA Langley Research Center in Virginia flew on the NASA's DC-8 aircraft during the NASA Genesis and Rapid Intensification Processes (GRIP) during the summer of 2010. The participation was part of the project Doppler Aerosol Wind Lidar (DAWN) Air. Selected results of airborne wind profiling are presented and compared with the dropsonde data for verification purposes. Panoramic presentations of different wind parameters over a nominal observation time span are also presented for selected GRIP data sets. The realtime data acquisition and analysis software that was employed during the GRIP campaign is introduced with its unique features.
\end{abstract}

Keywords: Wind profile algorithm, coherent lidar, GRIP, DAWN AIR

\section{INTRODUCTION}

There have been extensive studies with the ground-based coherent Doppler lidar system housed in the trailer test-bed VALIDAR (Validation Lidar) since 2000 [3-14]. The system is a high-pulsed energy, 2 micron, solid-state Doppler laser developed at NASA Langley Research Center in Hampton, Virginia, USA. The data acquisition system for VALIDAR requires a high data sampling rate, resulting in extensive data volume to be processed real-time. The next generation of such a lidar and data acquisition system has been developed for the project DAWN AIR and flew on the NASA's DC-8 airborne laboratory during the summer of 2010 in support of the NASA Genesis and Rapid Intensification Processes (GRIP) hurricane campaign [1-2]. The real-time data acquisition system is called DAPDS (Data Acquisition, Processing, and Display System) and executes its functionalities by running software called DAPS (Data Acquisition and Processing Software). This paper is to introduce the wind profiling algorithm concept in DAPS for the airborne lidar data acquired during the GRIP campaign and the examples of estimated wind parameters. A trend of wind profile over an extended period of observation is an important way of interpreting a collection of wind parameters. The samples of panoramic color display of selected wind parameters are also presented in this paper.

\section{PULSED 2-MICRON COHERENT DOPPLER LIDAR SYSTEM}

Doppler lidar works by measuring the shift in frequency of a pulse of laser light that is scattered by aerosols in the atmosphere. Aerosols are light enough to be suspended in the atmosphere, and their motion is an indicator of the wind field in which the aerosols are entrained. By steering the lidar beam in multiple azimuths, the wind vector can be determined. By use of a pulsed laser, wind can be determined as a function of range from the lidar by relating the timing of the outgoing laser pulse and atmospheric backscatter.

The lidar is based on 2- $\mu \mathrm{m}$ wavelength lasers that have been under development for many years at NASA Langley Research Center, most recently with the support of the NASA Earth Science Technology Office. A 2- $\mu \mathrm{m}$ wavelength is chosen for three reasons: eye safety, very low atmospheric clear air extinction, and availability of high energy pulsed laser output.

*Jeffrey.Y.Beyon@nasa.gov; phone 1757 864-4249; fax 1757 864-7944; www.nasa.gov 
A diode-pumped Ho:Tm:LuLiF laser forms the transmitter for the lidar, including an optical amplifier to boost the energy to $250-\mathrm{mJ}$ at $10-\mathrm{Hz}$ pulse repetition rate and 200-ns pulse width. The basic architecture of the lidar is shown in Figure 1. Details on the design of the laser and optical aspects of the lidar have been described in Koch et al. 2007 [9] and Koch et al. 2010 [3]. Work in recent years has been to harden the laser and optical design for immunity to vibration, temperature variation, and pressure variation encountered in an aircraft environment. Figure 2 shows the lidar mounted into the NASA DC-8 aircraft.

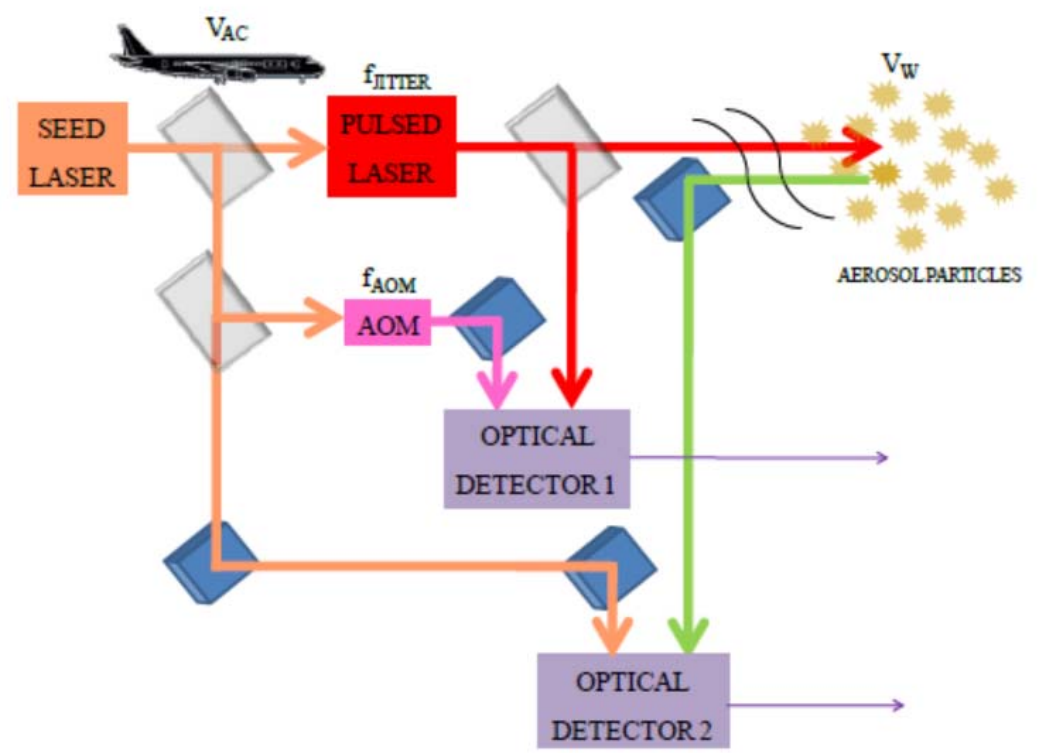

Fig. 1. Block diagram of laser and optical design.

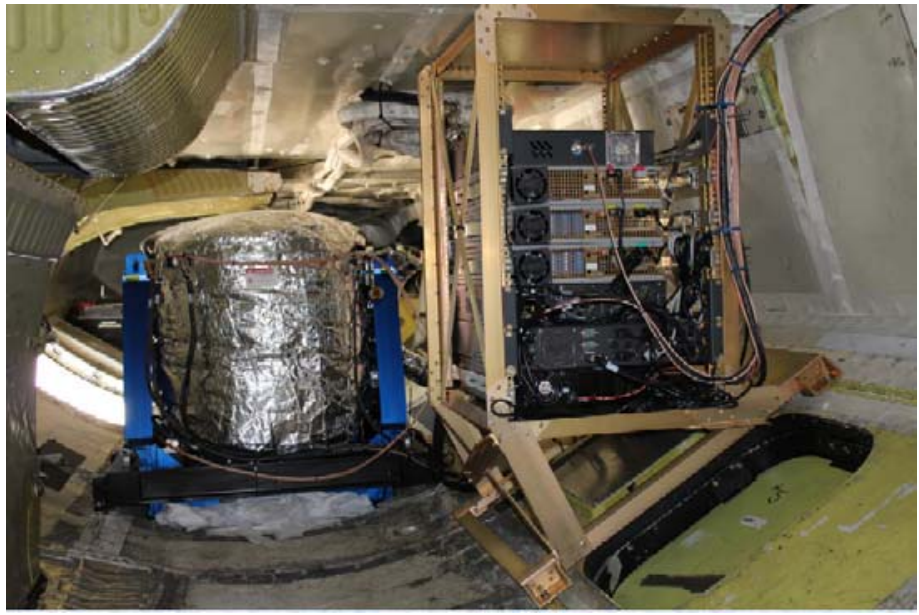

Fig. 2. The laser and optics are packaged in a cylindrical enclosure, shown here installed in the cargo bay of the DC-8 aircraft.

\section{HIGH-SPEED DATA ACQUISITION, PROCESSING, AND DISPLAY SYSTEM}

The high-speed DAPDS consists of the INS/GPS subsystem (IGSS), scanner control subsystem (SCSS), digitizer subsystem (DSS), and digital signal processing subsystem (DSPSS). In order to meet the system requirements such as $10 \mathrm{~Hz}$ trigger rate while acquiring and processing 55,000 samples at $500 \mathrm{MHz}$, all subsystems need to function in a 
rigidly controlled fashion. The DSPSS typically must be able to execute 200 - 300 512-point FFT computations without missing any external $10 \mathrm{~Hz}$ trigger pulse. All the subsystems reside in a 6U compact PCI chassis, and are controlled by DAPS written in ANSI C. In order to optimize the performance of DAPDS and its interaction with the subsystems, DAPS is carefully designed to acquire and process lidar data at the required speed while utilizing multi-threading and preventing memory leak situations.
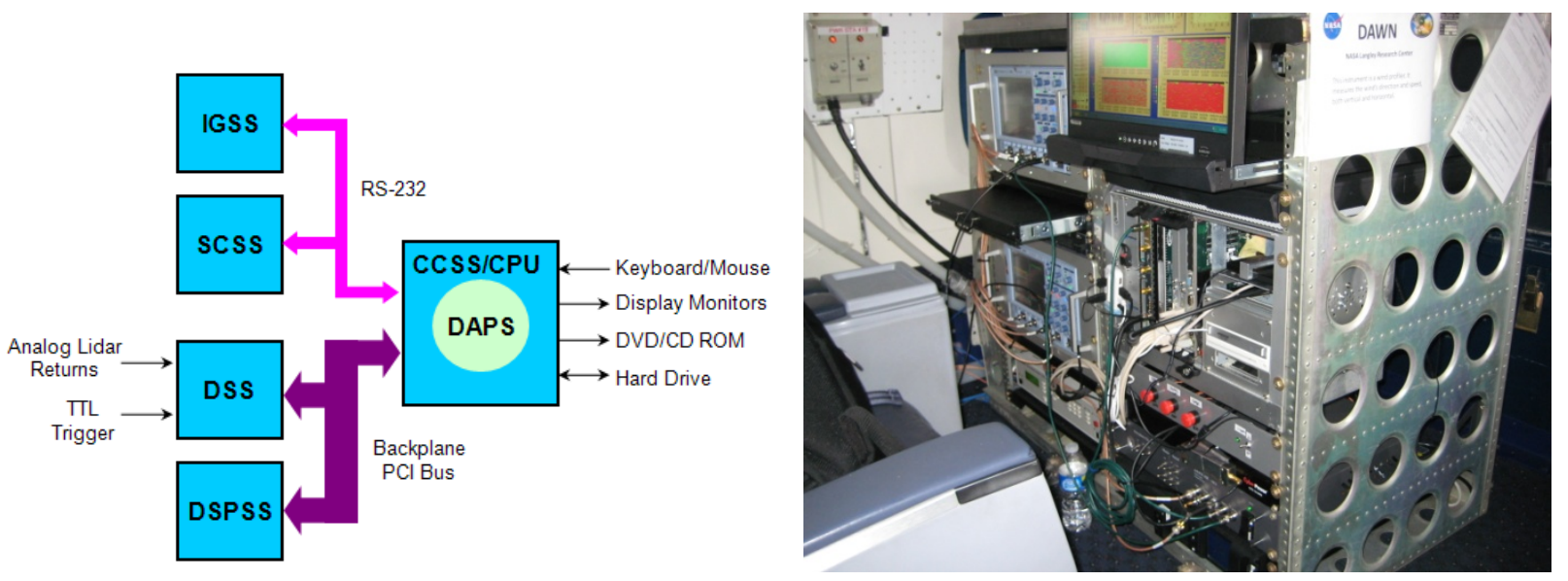

Fig. 3. Data Acquisition, Processing, and Display System (DAPDS) and the laser electronics system in the DC-8 during the mission GRIP

\section{WIND PROFILING ALGORITHM FOR AIRBORNE LIDAR DATA}

In order to process the airborne lidar data acquired during the GRIP campaign, a few different versions of new airborne lidar processing algorithms were developed. Additional analysis procedures compared to the ground-based wind profiling algorithm were necessary to account for the Doppler shifts due to the aircraft movement and its speed along with roll, pitch, and yaw displacements. Data processing results from one of the algorithms using two line-of-sights (LOSs) are shown in this paper.

\begin{tabular}{|c||c|c|}
\hline DAPS & Real-Time Version & Ground-Based Version \\
\hline \hline Programming Language & ANSI-C & LabVIEW \\
\hline Data Acquisition & Yes & No \\
\hline Scanner Control & Yes & No \\
\hline INS/GPS Control & Yes & 2-directional \\
\hline Wind Profiling Algorithm & 5-directional & Yes \\
\hline Dropsonde Data Comparison & No & Yes \\
\hline Noise Whitening & Yes & 512, 1024, 2048, 4096 \\
\hline FFT-Size & $512,1024,2048,4096$ & \\
\hline
\end{tabular}

Table 1. Comparison of two versions of wind profiling software for DAWN AIR.

DAPS is unique in many aspects. It is the first real-time airborne wind lidar data acquisition and processing software that runs in DAPDS controlling all the subsystems. Some of its unique features are the self-aware auto-recalibration capability of digitizer, the data interface feature with the INS/GPS subsystem, the faulty data acquisition auto-recovery feature, and the high speed real-time data acquisition and processing capabilities. DAPS runs several different versions of airborne wind profiling algorithms and successfully completed the GRIP campaign without an incident of crash or system malfunction during the approximately 130 hours of total flight time.

Figure 4 shows the abstract concept of wind profiling algorithm using airborne wind lidar data. The lidar returns from 
the Coherent Doppler lidar system get conditioned for digitization process. Using an external TTL trigger pulse, the DSS samples the conditioned lidar returns. The shaded area shows the data processing that occurs in DAPS. The digitized lidar return samples are divided into multiples of range gates or bins of the same size with user selected overlap. Once such segmentation is complete, the FFT of the range gate data in each bin is calculated simultaneously in DSPSS. During this spectral analysis processes, the FFT results are aligned across the range bins, and the background instrument noise is compensated by a noise whitening method, followed by peak detection. Once the peak frequency is identified, Doppler shift estimates are computed. Using the INS/GPS data, the effect of roll, pitch, and yaw of the aircraft is accounted for before the final wind parameters are estimated, which are displayed for users and archived in storage devices. The TTL trigger pulse rate is $10 \mathrm{~Hz}$, and the spectral analysis must be completed before the next TTL pulse arrives.

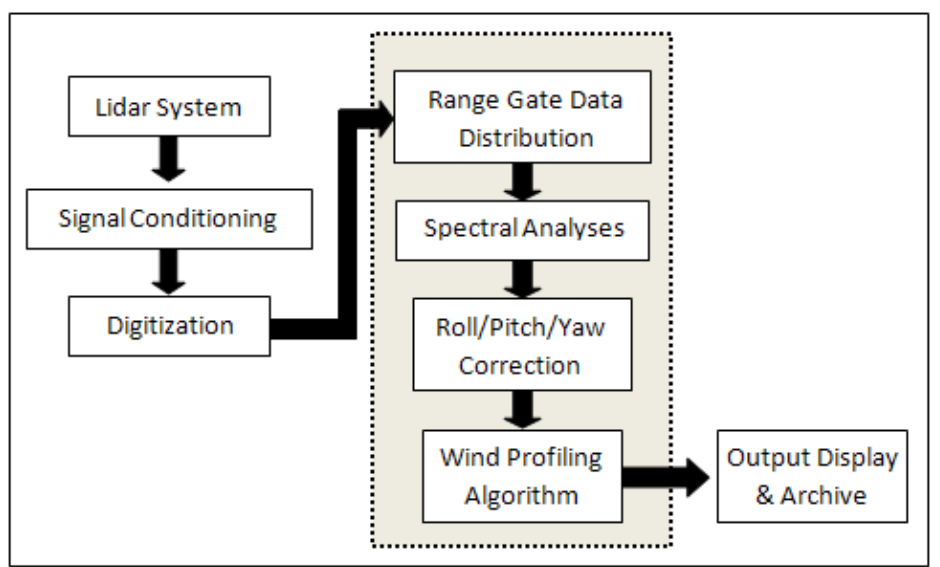

Fig. 4. Wind profiling data process procedures. Shaded area represents the data processing in DAPS.

Figure 5 shows the power distribution and the Doppler shift straight ahead of the aircraft. Figures 6 through 8 show the horizontal wind speed (HWS) and the horizontal wind direction (HWD) for two different FFT sizes: 512 and 2,048. The data used for this comparison were acquired at 17:20:11 Zulu on September 1, 2010. The x-axis in Figure 5 and the altitude in Figures $6-8$ are reversely related. The LOS range is measured from the aircraft and away, and the altitude from the ground vertically toward the aircraft. This means $0 \mathrm{~km}$ LOS range is at the aircraft, and $0 \mathrm{~km}$ altitude is on the ground. It is shown in Figure 5 that the signal-to-noise-ratio (SNR) is higher near the aircraft and the ground, and consequently we have poor HWS and HWD measurements in 4.5-9 km altitude as shown in Figures 6 through 8.

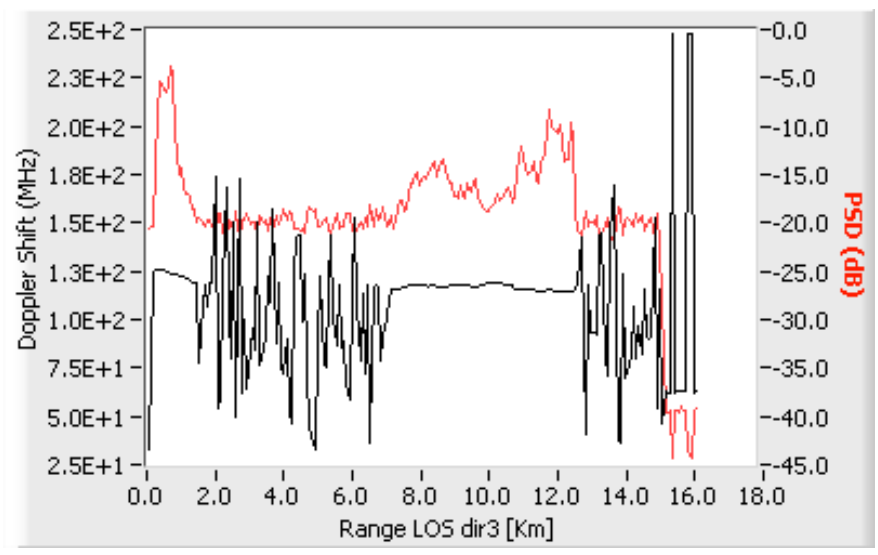

Fig. 5. Power distribution and Doppler Shift at LOS3. Range LOS $0 \mathrm{~km}$ is at the aircraft and becomes larger as moving away from the plane. 
The results of DAWN AIR airborne wind profiling algorithm are compared with the dropsonde measurements in Figures 6 - 8. A dropsonde is a weather probing device designed to be dropped from a airplane, and it transmits weather measurements as it falls until it reaches the ground. Note that a comparison in an identical environment condition is not possible. The lidar wind profiling is almost instantaneous, whereas the dropsonde measurements are over an extensive period of time until the dropsonde reaches the ground. The fall path of dropsonde is not straight and is affected by winds. Even though the dropsonde data reveal the wind profile over a period of time and its drop path does not align with the LOS of the DAWN AIR Doppler lidar, we compare both results as one of validation methods. Note that the HWD is extremely sensitive to the accuracy of algorithm, and it is clearly shown that DAWN AIR airborne wind profiling algorithm returns HWDs closely matching those from dropsonde measurements.

Using a larger size FFT via zero padding, Figure 7 shows more details of HWS. In Figure 8, the comparison of different FFT sizes without the dropsonde data is depicted. It clearly shows the benefit of larger size FFT via zero padding where more details of HWS are revealed. The advantage of zero padding in HWD estimates, however, is not as significant as that in HWS estimates.
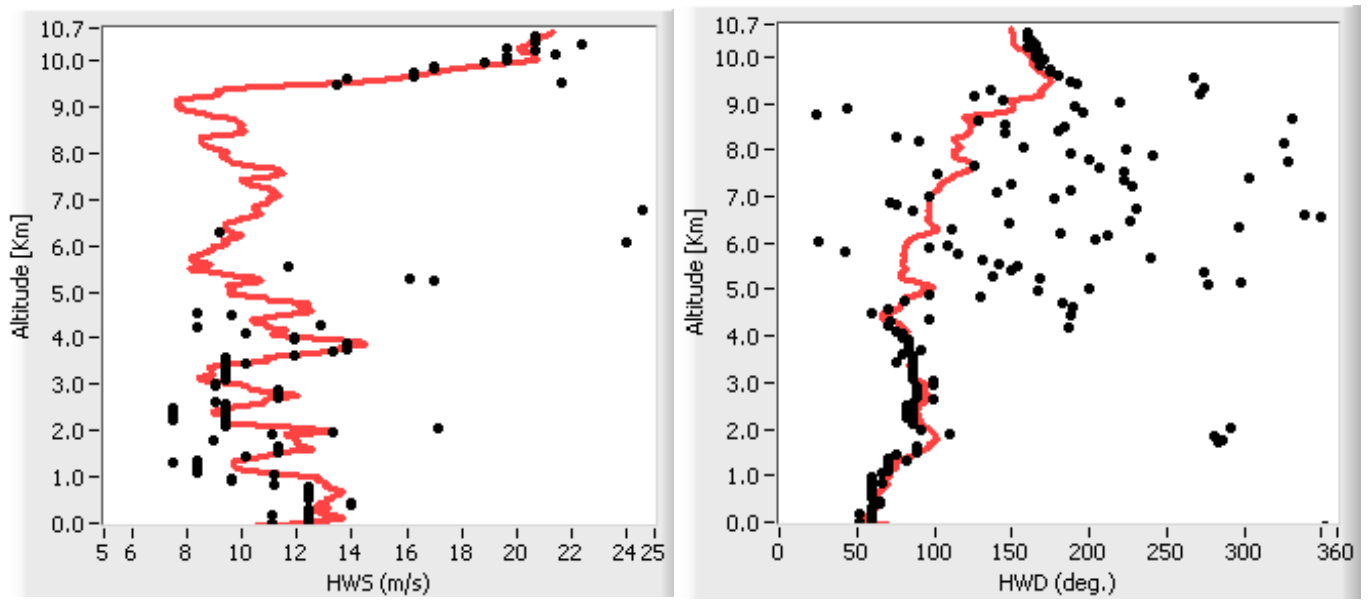

Fig. 6. Horizontal Wind Speed (HWS) and Horizontal Wind Direction (HWD). FFT size $=512$. Line $=$ Dropsonde, Dots $=$ DAWN AIR .
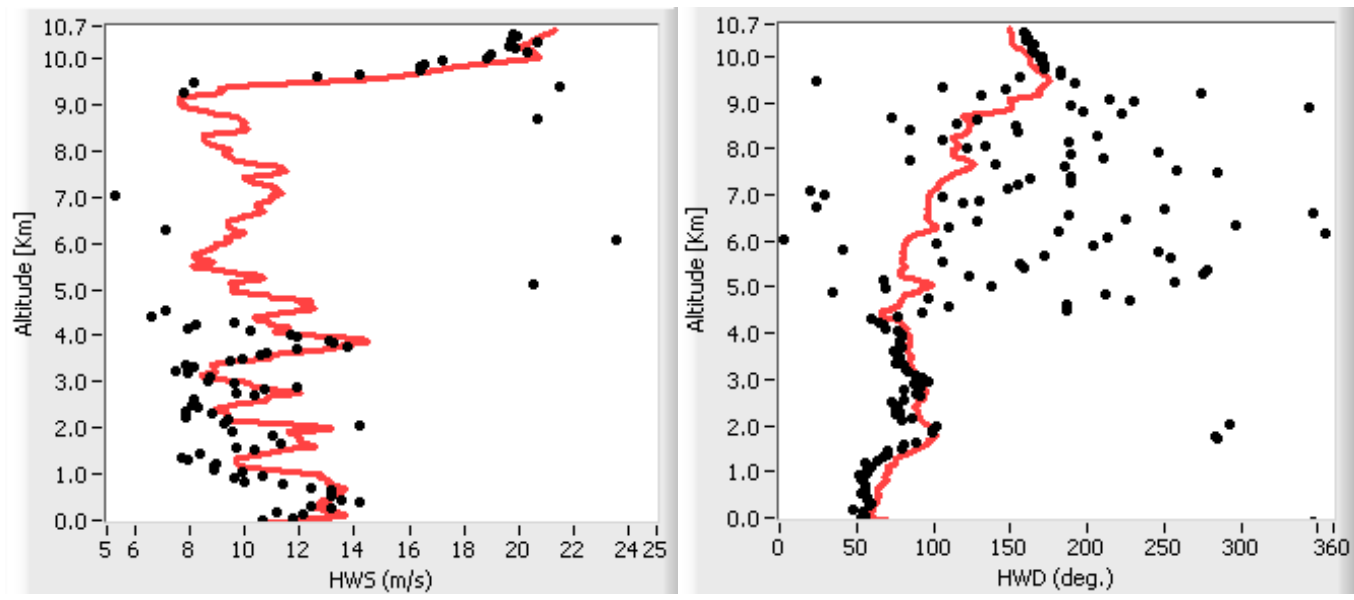

Fig. 7. Horizontal Wind Speed (HWS) and Horizontal Wind Direction (HWD). FFT size $=2048$. Line $=$ Dropsonde, Dots $=$ DAWN AIR . 

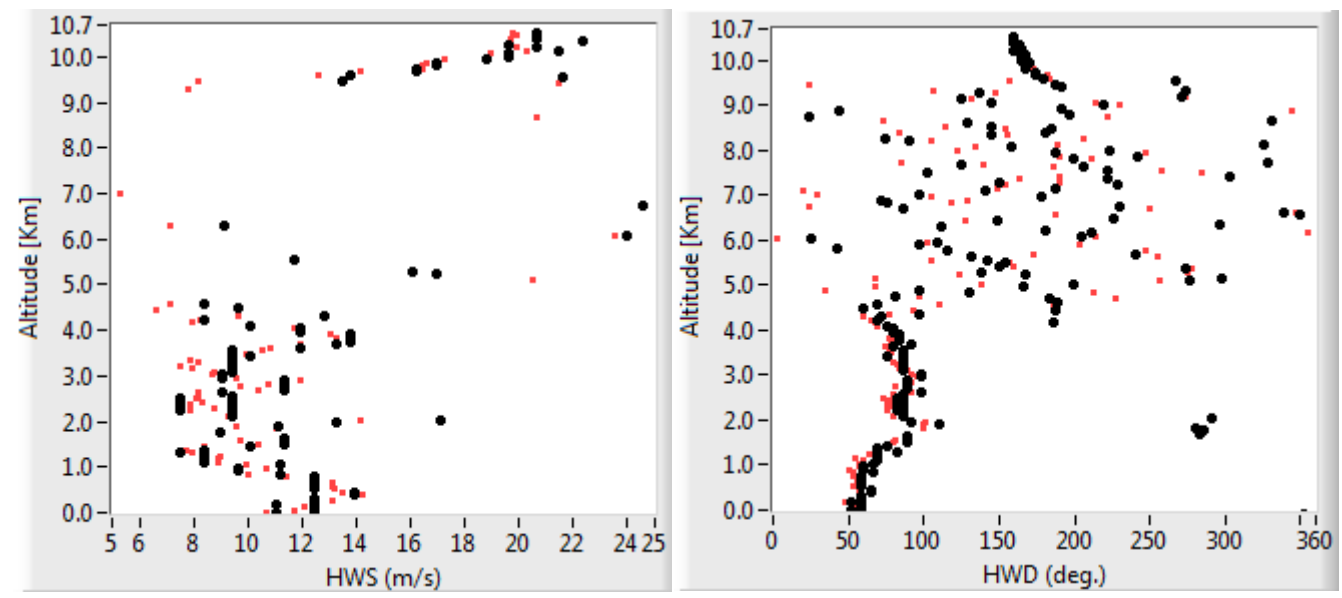

Fig. 8. Comparison of FFT size in HWS and HWD from DAWN AIR. Smaller square dots $=2048$, Larger circle dots $=512$.

The panoramic display of wind parameters over an extensive observation period is useful to show wind patterns. Figure 9 shows the power distribution in $\mathrm{dB}$ over about an hour of observation time span on September 1, 2010. It is the indication of aerosol distribution that can sometimes be understood as cloud distributions. When the DC-8 was flying over a land (hard surface), the power display was showing the contour of the land near zero altitude. Figures 10 and 11 similarly show the long haul patterns of horizontal wind speed and direction using color to indicate the strength in $\mathrm{m} / \mathrm{s}$ and the bearing in degree.
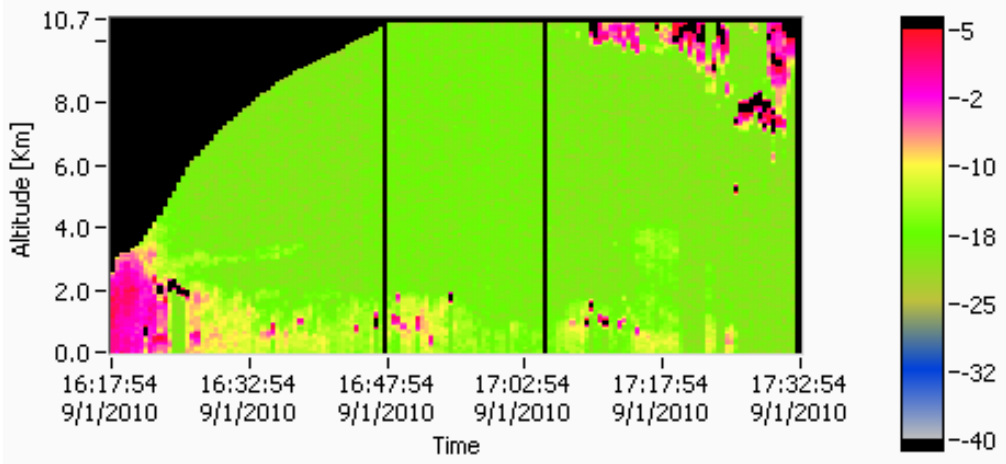

Fig. 9. Panoramic display of power distribution in $\mathrm{dB}$.

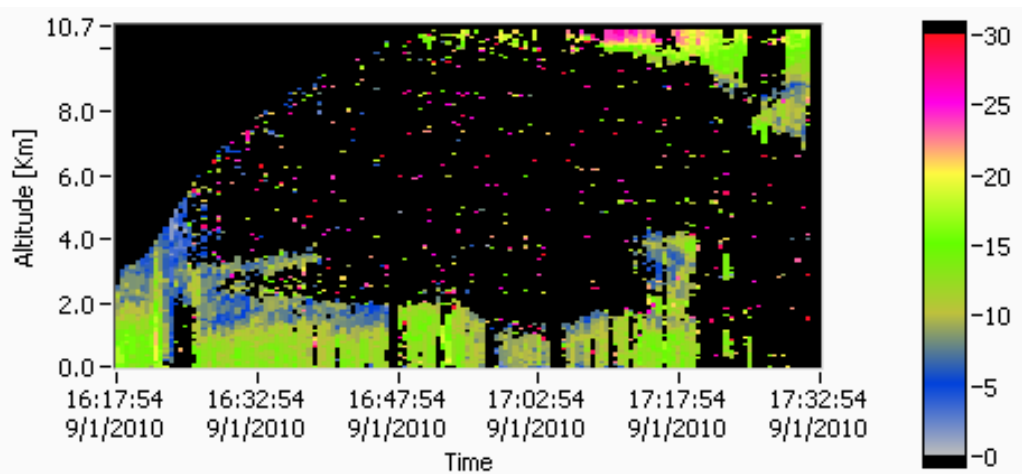

Fig. 10. Panoramic display of HWS [m/s]. 


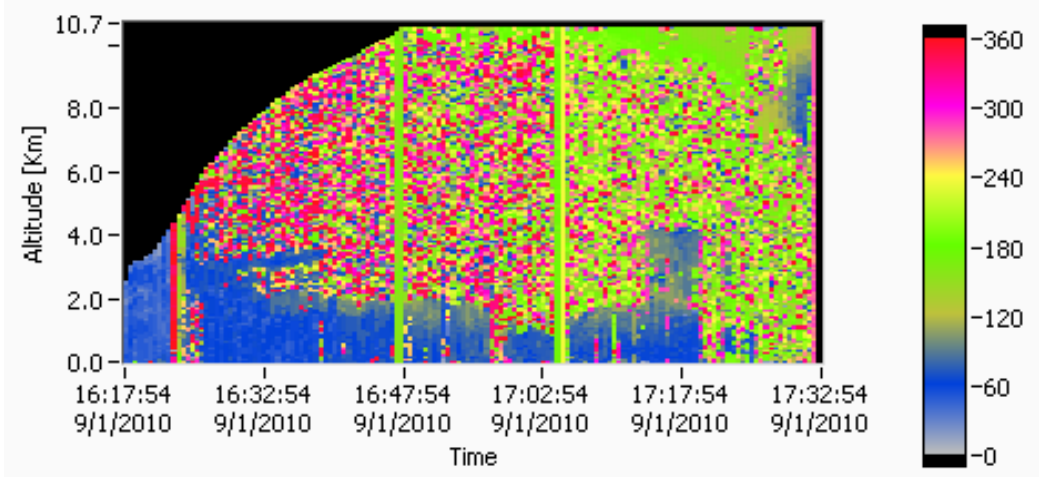

Fig. 11. Panoramic display of HWD [degree].

\section{CONCLUSION}

The airborne wind profiling algorithm for DAWN AIR lidar system is introduced. There are a few different versions, and the one using two LOS directions is presented in this paper. It currently runs in LabVIEW, and will be implemented in DAPS in a near future. It is the first airborne wind profiling algorithm using DAWN AIR airborne Doppler wind lidar data. DAPS is a real-time data acquisition and processing software that runs in DAPDS. The most unique feature of DAPDS and DAPS is the real-time data processing capability while executing high-speed data acquisition. The amount of wind lidar data acquired for each TTL trigger pulse is large such as 50,000 in order to cover a long range of wind field, and both DAPDS and DAPS are well equipped and designed to handle such a case. We have shown the advantage of larger FFT size in spectral analysis, and the trade-off will be slower processing speed. The results of the DAWN AIR airborne wind profiling algorithm was compared with dropsonde data and close match in high SNR altitudes validates the accuracy of the new algorithm. Another version of algorithm using all five LOSs is currently in the works.

\section{ACKNOWLEDGMENT}

The authors are grateful for support from NASA Science Mission Directorate (SMD), the SMD Airborne Instrument Technology Transition program, the NASA SMD Earth Science Technology Office (ESTO) Instrument Incubator Program, the ESTO Laser Risk Reduction Program, Langley Research Center (LaRC), and the LaRC Engineering Directorate.

\section{BIBLIOGRAPHY}

[1] J. Y. Beyon, G. J. Koch, and M. J. Kavaya "Development of the Data Acquisition and Processing System for a Pulsed 2-Micron Coherent Doppler Lidar System,” in Proc. of the SPIE Asia-Pacific Remote Sensing (7860-9), Incheon, Republic of Korea, October, 2010.

[2] J. Y. Beyon, G. J. Koch, and M. J. Kavaya "Data Acquisition and Processing System for Airborne Wind Profiling with a Pulsed, 2-Micron, Coherent-Detection, Doppler Lidar System,” in Proc. of the Earth Science Technology Forum 2010, Arlington, VA, June, 2010.

[3] G.J. Koch, J.Y. Beyon, P.J. Petzar, M. Petros, J. Yu, B.C. Trieu, M.J. Kavaya, U.N. Singh, E.A. Modlin, B.W. Barnes, and B.B. Demoz, "Field Testing of a High-Energy 2-um Doppler Lidar,” Journal of Applied Remote Sensing 4, 043512, 2010.

[4] K. Vermeesch, G. Koch, B. Gentry, T. Bacha, H. Chen, and B. Demoz, "Comparisons of Ground-Based, Radiosonde, and Aircraft Wind Measurements at the Howard University Beltsville Research Site,” Wind Lidar Working Group Meeting, June, 2009. 
[5] J. Y. Beyon, G. J. Koch, M. J. Kavaya, and M. Sahota, "Comparison of theoretical and empirical statistics of wind measurements with validation lidar (VALIDAR)," in Proc. of the Defense and Security Symposium 2008(6968-59), Orlando, FL, March, 2008.

[6] J. Y. Beyon, G. J. Koch, M. J. Kavaya, and M. Sahota, "Comparison of theoretical and empirical statistics of wind measurements with validation lidar (VALIDAR)," in Proc. of the Defense and Security Symposium 2008(6968-59), Orlando, FL, March, 2008.

[7] J. Y. Beyon and G. J. Koch, "Novel nonlinear adaptive Doppler-shift estimation technique for the coherent Doppler validation lidar,” Optical Engineering, Vol. 46, No. 1, pp. 016002-1 - 016002-10, January 2007.

[8] J. Y. Beyon, G. J. Koch, and S. Ismail "Signal processing techniques for heterodyne differential absorption lidar," in Proc. of the Defense and Security Symposium 2007 (6567-53), Orlando, FL, April, 2007.

[9] G.J. Koch, J.Y. Beyon, B.W. Barnes, M. Petros, J. Yu, F. Amzajerdian, M.J. Kavaya, and U.N. Singh, "HighEnergy 2- $\mu m$ Doppler Lidar for Wind Measurements," Optical Engineering, Vol. 46(11), pp. 116201-1 116201-14, November, 2007.

[10] J. Y. Beyon and G. J. Koch, "Novel Nonlinear Adaptive Doppler Shift Estimation Technique (NADSET) for the Coherent Doppler Lidar System VALIDAR,” in Proc. of the Defense and Security Symposium 2006 (62361), Orlando, FL, April, 2006.

[11] J. Y. Beyon and G. J. Koch, "Resolution Study of Wind Parameter Estimates by a Coherent Doppler Lidar System,” in Proc. of the Defense and Security Symposium 2006 (6214-3), Orlando, FL, April, 2006.

[12] J. Y. Beyon and G. J. Koch, "Wind Profiling by a Coherent Doppler Lidar System VALIDAR with a Subspace Decomposition Approach," in Proc. of the Defense and Security Symposium 2006 (6236-5), Orlando, FL, April, 2006.

[13] J. Y. Beyon, G. J. Koch, and Z. Li "Noise Normalization and Windowing Functions for VALIDAR in Wind Parameter Estimation," in Proc. of the Defense and Security Symposium 2006 (6214-4), Orlando, FL, April, 2006.

[14] J. Yu, B.C. Trieu, E.A. Modlin, U.N. Singh, M.J. Kavaya, S. Chen, Y. Bai, P.J. Petzar, “1 J/pulse Q-switched 2 um solid-state laser," Optics Letters 31, 462-462, 2006. 